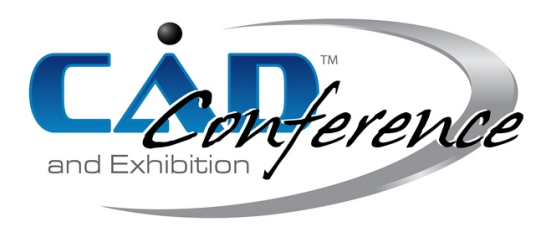

Title:

\title{
Modeling Coverage Areas of Anisotropic Transmitters by Voronoi-like Structures
}

Authors:

Martin Held, held@cs.sbg.ac.at, Salzburg University, Austria

Peter Palfrader, palfrader@cs.sbg.ac.at, Salzburg University, Austria

Keywords:

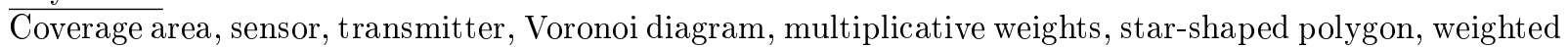
straight skeleton

\section{DOI: $10.14733 /$ cadconfP.2021.283-287}

\section{Introduction:}

Consider a set $S$ of points in the plane, called sites, and a signal that is sent out from each site. Now assume that each signal starts at the same time, say time $t:=0$, and propagates with unit speed uniformly in all directions. The locations at time $t \geq 0$ that are reached by a signal sent out from a site $s \in S$ is given by a circle ("offset circle") of radius $t$ centered at $s$, and the area that has been covered by that signal by time $t$ is the corresponding circular disc.

For $t$ sufficiently small, no pair of these discs will intersect. However, as $t$ increases, intersections will occur. Apparently, intersections of two such circles correspond to points of the plane that are reached by two different signals at the same time. Assigning each locus of the plane to the site whose signal reached it first yields a partition of the plane that is well-known as the Voronoi diagram of $S$; cf. Fig. 1(a). Adjacent regions of this partition are separated by straight-line segments. (We refer to the textbook [11] for more background information on Voronoi diagrams.)

The boundary of the area covered by at least one signal by time $t$ is called the wavefront of $S$ at time $t$. It is easy to see that every wavefront of $S$ consists of circular arcs whose endpoints lie on the Voronoi diagram of $S$.

Voronoi diagrams can be generalized to settings where the signals no longer all travel at the same speed. To each site $s$ a weight $\sigma(s)$ is assigned that specifies how fast the signal travels: In this modified setting, the signal has reached points at distance $\sigma(s) \cdot t$ (from $s$ ) at time $t$. The corresponding Voronoi diagram is known as multiplicatively weighted Voronoi diagram [5]. The common boundary of two adjacent regions is no longer a line segment but is a circular arc. Also, the region associated with a specific site $s$ can now be disconnected or multiply-connected; cf. Fig. 1(b). In a similar way, one can generalize the Voronoi diagram by allowing the sites to start emitting their signals at different points in time. This leads to the concept of additively weighted Voronoi diagrams.

Voronoi diagrams have become an important geometric tool for modeling and analyzing coverage areas of sensors and transmitters. We refer to $[4,10,12]$ for sample publications on this application. Common to these publications is the fact that the signal propagation is assumed to be uniform both over all sites and over all directions for each site. 


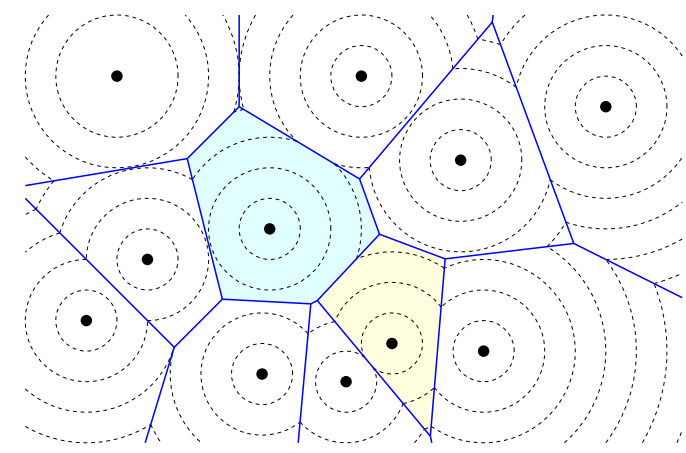

(a)

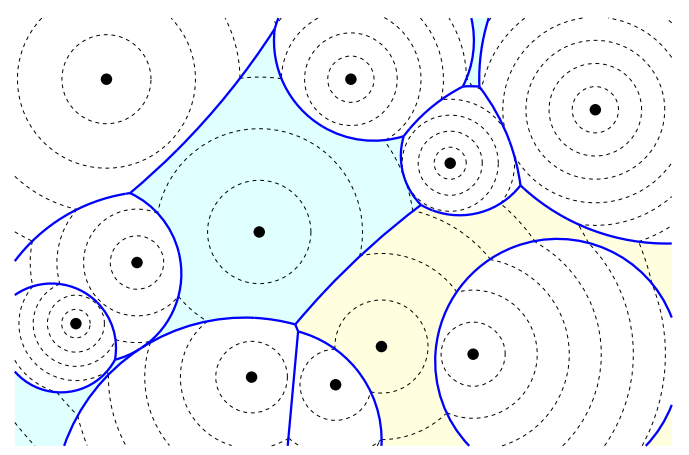

(b)

Fig. 1: (a) The Voronoi diagram of point sites. Wavefronts for some specific points in time are shown by dashed curves. (b) The multiplicatively weighted Voronoi diagram has regions which need not be connected (e.g., the light-blue region). Again, some wavefronts are shown by dashed curves.

\section{Our Contribution:}

The assumption that signals and their spreading are uniform provides only a rather coarse approximation of reality. Rather, different sites should be assumed to emit signals of different strengths. Furthermore, the spreading of a signal should be assumed to be anisotropic, i.e., to vary with the direction.

In this work we show how concepts of computational geometry can be applied to provide a refined model for a subsequent coverage analysis: We interpret increasing distance in the Voronoi setting as decreasing signal strength and provide a system where initial signal strength may vary among the sites and its rate of change may vary over different directions for each site. Depending on whether or not the spreading of a signal is stopped once it reaches a point of the plane that has already been covered by some other signal, we get connected or disconnected coverage areas.

Star-induced Voronoi diagram:

In order to model an anisotropic spreading of signals, we consider a variant of point-site Voronoi diagrams: We no longer use an offset circle to model the area covered by one site's signal. Rather, we replace the circle by a star-shaped polygon that contains the site in its kernel. (Recall that a polygonal area is star-shaped if it contains at least one point from which its entire boundary is visible; the set of all those points is called its kernel.) We call such a polygon an offset star. Mitered offsets of an offset star are the appropriate generalization of the expansion of offset circles; cf. Fig. 2: If a vertex $v$ of the offset star of $s$ at time $t$ is at distance $t \cdot d$ from $s$, for some $d \geq 0$, then it will be at distance $t^{\prime} \cdot d$ from $s$ at time $t^{\prime}$, thereby also moving on the ray from $s$ through $v$. Of course, we allow offset stars of different shapes and sizes for different sites. And, in an analogy to additively weighted Voronoi diagrams, we allow the offset stars to start their expansion at different points in time.

One way to interpret this generalization is that each input site is the location of a transmitter whose signal strength decays with distance, but not at the same rate in every direction. Then, offset stars of the same shape but at different sizes can be seen as transmitters with the same anisotropic emission characteristics but whose signals decay at different rates. Furthermore, the wavefronts derived from the expansion of all offset stars are iso-contours of signal strength.

In theory, any star-shaped polygon that contains its site in its kernel can be used as offset star for that site. Of course, the more vertices the polygon has, the finer a direction-dependence of the spreading of the signal can be modeled. Feedback obtained from companies tells us that polygons with 10-20 vertices will be good enough for practical applications. Note that the offset stars shown in this work (cf. Fig. 2) were chosen for visual clarity and simplicity rather than genuine practical relevance. 


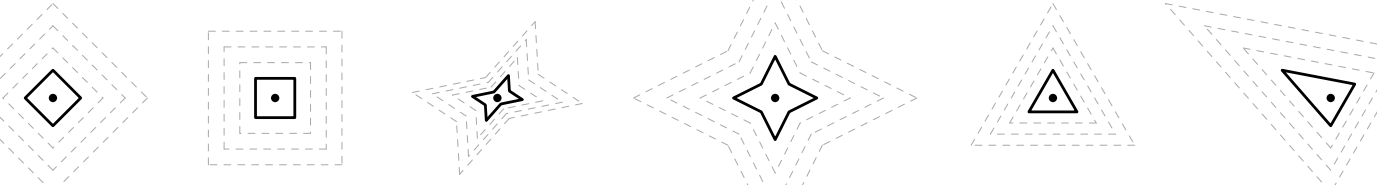

Fig. 2: Six sites with different offset stars are shown. Some mitered offsets for distinct points in time show the expansion of the offset stars, i.e., how the signals spread in different directions.

Similar to how classic Voronoi diagrams tessellate the plane into regions such that all points within the same region have been reached first by the same offset circle, we now want to partition the plane into regions such that all points of a region are reached first by the same offset star. We call this structure the star-induced (weighted) Voronoi diagram; cf. Fig. 3(a). As for standard multiplicatively weighted Voronoi diagrams, some regions may be disconnected and consist of more than one face. For instance the purple region of the site close to the bottom-right corner of Fig. 3(a) consists of two faces. (Several more disconnected components show up outside of the image frame.)

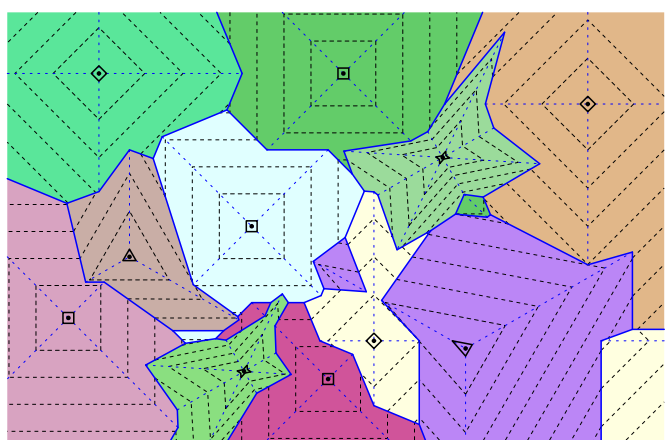

(a)

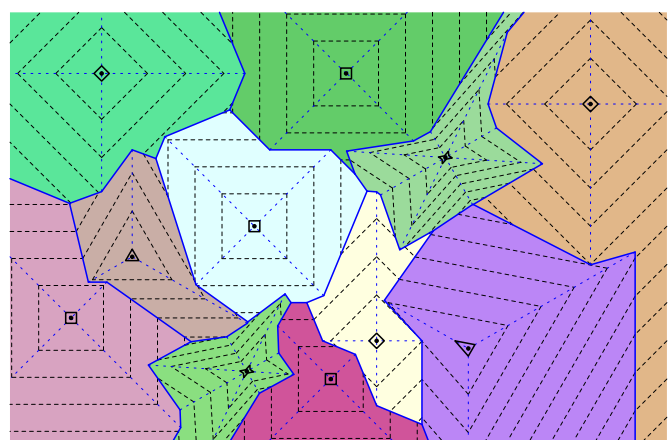

(b)

Fig. 3: (a) A sample star-induced Voronoi diagram for the sites shown in Fig. 1. Each region is assigned a unique color and its offset star is shown. Dashed blackdashed lines show a family of wavefronts. (b) A sample star-induced skeleton for the same sites. Dashed blue lines are straight-skeleton arcs that are removed in the post-processing step, solid blue lines are the final edges tessellating the planes into distinct regions, with one connected region per input site. A family of wavefronts is shown for the same points in time as in (a).

Computing a star-induced Voronoi diagram: For the sake of descriptional simplicity, we start with assuming that all additive weights are zero, i.e., that all signals start to spread at the same time. All offset stars are scaled uniformly such that no pair of offset stars overlaps at time $t:=1$. (This can be done easily based on the standard Voronoi diagram of the sites.)

We will now take a look at how that portion inside of the wavefront that belongs to a site's region changes as time progresses. The spreading of the signals starts at time $t:=0$. At this time, the signal of each transmitter has not yet spread at all. Hence, at this time, each site's region within the wavefront only consists of the site itself. By our assumption, at time $t:=1$ no two signals have yet interfered, and each site's signal has covered its corresponding offset star. Of course, intersections of the offset stars will occur as time progresses.

To get a hand on these intersections and obtain an actual algorithm for constructing a star-induced 
Voronoi diagram, we lift the entire problem into $\mathbb{R}^{3}$, where the input plane (of $\mathbb{R}^{2}$ ) is the $x y$-plane and the third coordinate represents the time $t$. Each site's signal is represented by one upside-down, infinitely tall, right pyramid whose apex at $t:=0$ coincides with the site's location. The inclinations of the lateral faces of the pyramid are chosen such that the intersection of the pyramid with the plane $t=1$ matches exactly the boundary of the offset star. (The intercept theorem implies that the intersection of the pyramid with some other plane parallel to the $x y$-plane yields a polygon that is a mitered offset of that offset star.)

Theory knows of a fairly general connection between Voronoi diagrams in $\mathbb{R}^{d}$ and lower envelopes of suitable distance functions in $\mathbb{R}^{d+1}$, see [6], which is also applicable to our problem: The lower envelope of those pyramids projected to the $x y$-plane or, equivalently, their so-called minimization diagram, yields our star-induced Voronoi diagram.

One can show that a delay in the start time of the signal of site $s$ can be handled by lifting the pyramid of $s$ vertically upwards above $s$ : If it is to start at time $t^{\prime}$, then its apex lies (vertically above $s$ ) in the plane $t=t^{\prime}$. No other modification of this general scheme is needed.

Implementation: We have developed a proof-of-concept implementation of this approach using exact arithmetic, based on CGAL and, in particular, based on CGAL's 3D Envelopes package [9]. To make it easier to use existing CGAL code, we chose to use finite triangles as the lateral faces of our pyramids instead of infinitely large ones. Of course, this raised a new problem: How tall is "sufficiently tall" to get the correct lower envelope and, thus, also the star-induced Voronoi diagram?

To obtain an upper bound we proceed as follows. First, we consider the supporting lines of all the offset stars' edges as they move away from their respective stars. At some point in time each supporting line will have left the bounding box $B$ of the input sites. If we make all pyramids at least this tall, then every pyramid will cover all of $B$, and the union of the pyramids' lateral faces projected to the $x y$ plane will form a star-shaped polygon with $B$ in its kernel. This implies that the minimization diagram restricted to $B$ is guaranteed to represent the Voronoi diagram restricted to $B$. However, using pyramids of that size does not guarantee that the minimization diagram correctly represents the Voronoi diagram outside of $B$, even in places where it is defined.

Therefore, as a second step, we also attempt to find the latest point in time when a reflex vertex of the mitered offset of an offset star pierces the supporting plane of any other pyramid face. After this time we know that the boundary of the minimization diagram will not see new vertices appear even if we proceed further in time. (There still might be changes as edges of the boundary vanish in edge events but if need be, these can be dealt with easily.)

We emphasize that the question of how tall the pyramids need to be, respectively how far in time one has to go with the wavefront propagation, is mostly academic. In practice, we are almost certainly given a region $R$ of interest for which we are to compute the diagram. And this is easily achieved, since it suffices to ensure that $R$ is covered by the projection of each pyramid. Then we can compute the minimization diagram. And in theory, the algorithms to compute lower envelopes [1] work just fine with infinitely large surfaces, and implementational convenience is of no concern, anyway.

\section{Star-induced skeleton:}

In the star-induced Voronoi diagram from the previous section, signals are sent out from sites and then they spread across the plane without affecting each other: Recall that the star-induced Voronoi diagram may contain disconnected regions, which implies that one signal had to travel over an area already covered by some other signal. Now we consider a second variant in which the signals do not overlap with each other. Again, we want to partition the plane into regions according to which site's signal reached a location first. But a signal stops to spread once it reaches a point that has already been covered by some other signal. This yields a partition of the plane where no region is disconnected. We call it the star-induced skeleton; cf. Fig. 3(b). 
It can be obtained by applying the theory of weighted straight skeletons $[2,3,8]$ of planar straight-line graphs. In particular, an extended version of a straight-skeleton algorithm by Aichholzer and Aurenhammer [2] was implemented recently by the authors' group at Salzburg [7]. It is called Surfer2. We use SURFER2 and combine it with some pre- and post-processing to obtain the star-induced skeleton.

As in the previous section, consider a set of offset stars that do not intersect pairwise. We note that the propagation speed of an edge of an offset star is proportional to the orthogonal distance of the edge's supporting line to the corresponding site. This defines a weight for every edge of every offset star. We use the weighted edges of all offset stars as input for SURFER2 to compute a straight skeleton of all offset stars. In a post-processing step we then merge straight-skeleton faces that come from different edges of the same offset star, thus obtaining our star-induced skeleton.

Source code: Our proof-of-concept code is provided on GitHub and can be used freely under the GPL(v3) license: https://github.com/cgalab/stardist/.

Acknowledgements: This work was supported by the Austrian Science Fund (FWF): Grant P31013.

\section{References:}

[1] Agarwal, P. K.; Schwarzkopf, O.; Sharir, M.: The Overlay of Lower Envelopse and Its Applications. Discrete Comput. Geom., 15(1), 1996, 1-13. https://doi.org/10.1007/BF02716576.

[2] Aichholzer, O.; Aurenhammer, F.: Straight Skeletons for General Polygonal Figures in the Plane. Voronoi's Impact on Modern Sciences II, volume 21. Institute of Mathematics of the National Academy of Sciences of Ukraine, Kiev, 1998, 7-21.

[3] Aichholzer, O.; Aurenhammer, F.; Alberts, D.; Gärtner, B.: A Novel Type of Skeleton for Polygons. J. Univ. Comp. Sci, 1(12), 1995, 752-761. https://doi.org/10.1007/978-3-642-80350-5_65.

[4] Argany, M.; Mostafavi, M. A.; Karimipour, F.: Voronoi-Based Approaches for Geosensor Networks Coverage Determination and Optimisation: A Survey. 7th Int. Symp. Voronoi Diagrams in Science \& Engineering. 2010, 115-123. https://doi.org/10.1109/ISVD.2010.36.

[5] Boots, B. N.: Weighting Thiessen Polygons. Economic Geography, 56(3), 1980, 248-259. https: //doi.org/10.2307/142716.

[6] Edelsbrunner, H.; Seidel, R.: Voronoi Diagrams and Arrangements. Discrete Comput. Geom., 1(1), December 1986, 25-44. https://doi.org/10.1007/BF02187681.

[7] Eder, G.; Held, M.; Palfrader, P.: On Implementing Straight Skeletons: Challenges and Experiences. 36th Int. Sympos. Comp. Geom. (SoCG 2020), LIPIcs, volume 164. 2020, 38:1-38:16. https://doi. org/10.4230/LIPICs. SoCG.2020.38.

[8] Eppstein, D.; Erickson, J.: Raising Roofs, Crashing Cycles, and Playing Pool: Applications of a Data Structure for Finding Pairwise Interactions. Discrete Comput. Geom., 22(4), 1999, 569-592. https://doi.org/10.1007/PL00009479.

[9] Halperin, D.; Meyerovitch, M.; Wein, R.; Zukerman, B.: 3D Envelopes. CGAL User and Reference Manual. CGAL Editorial Board, 5.2 edition, 2020. https://doc.cgal .org/5.2/Manual/packages. html\#PkgEnvelope3.

[10] Megerian, S.; Koushanfar, F.; Potkonjak, M.; Srivastava, M. B.: Worst and Best-case Coverage in Sensor Networks. IEEE Trans. Mobile Comput., 4(1), 2005, 84-92. https://doi.org/10.1109/ TMC. 2005.15.

[11] Okabe, A.; Boots, B.; Sugihara, K.; Chiu, S. N.: Spatial Tesselations: Concepts and Applications of Voronoi Diagrams. Wiley, 2nd edition, 2000. ISBN 978-0471986355.

[12] Wang, Q.; Guo, G.; Cao, L.; Xing, X.: Voronoi Coverage Algorithm Based on Connectivity for Wireless Sensor Networks. 34th Chinese Control Conference. 2015, 7833-7837. https://doi.org/ 10.1109/ChiCC.2015.7260884. 